enthusiasm for teaching, but optimising the student experience is crucial, so novel ways of controlling quality must be sought.

The results of this survey will be used to inform changes to this particular teaching programme such as increasing the use of role-play teaching and emphasising the importance of structured firm teaching, with regular consultant tutorials as well as sessions with junior doctors. The findings could also inform adjustments to psychiatric teaching programmes at other institutions. More studies examining the specific components of undergraduate teaching programmes in psychiatry are required to establish which teaching methods students find most stimulating and which aspects need improvement. Shaping teaching programmes in this way may improve the overall undergraduate experience of psychiatry for students and perhaps even help recruitment into the specialty.

1 Simmons $M$, Wilkinson P. Lectures versus case discussions: randomised trial of undergraduate psychiatry teaching. Psychiatrist 2012; 36: 146-50.

Kamran Ahmed, ST6 psychiatry, South London and Maudsley psychiatric training scheme, email: kamranahmed_786@hotmail.com; Jessica Makey, medical student, King's College London Medical School, Francesca Eddy, medical student, King's College London, Bronwen Deacon, medical student, King's College London.

doi: 10.1192/pb.36.7.271a

\section{The psychiatry experience from a medical student perspective}

I am a third-year medical student in the last week of psychiatry rotation. Although many positives emerged from this experience of psychiatry, it is clearly useful to identify areas of weakness, as a good undergraduate experience is crucial to encouraging recruitment into the profession.

The first challenge facing my curriculum is from sharing timetable space with neurology in a 'brain-and-mind' rotation. It is perhaps an indictment of attitudes towards mental health that psychiatry is found in this position, something which is not required of my other third-year rotations. The very title 'brain and mind' is fatally misleading, insidiously suggesting that neurology is the 'brain' (i.e. the challenging, scientific area), whereas psychiatry is relegated to the 'mind' (and by association, the opposite) by medical school and students alike. I have observed the damage to the attitudes of students previously sanguine towards psychiatry originating from this false and simplified dichotomy.

With psychiatry being the Cinderella of the 'brain and mind' rotation, the contrast with the 'brain' of neurology is stark. Neurology lectures are delivered by a locally eminent neurologist, whereas a majority of the psychiatry lecture curriculum is delegated to nurses trained in medical education. I cannot be alone in suspecting that it would be considered unthinkable for the neurology component to be delivered by nurses, yet somehow this attitude is acceptable and pervasive in psychiatric undergraduate education. Part of a wider stigma, perhaps? That, of course, is not a criticism of the teaching delivered by the psychiatric nurses (and the multidisciplinarian approach is vital in psychiatry), but if attitudes (and therefore recruitment) are to improve among medical students, then it is essential that psychiatrists lead the taught curriculum. Not only would this potentially raise standards, but also provide students with psychiatric role models. Most can recall doctors or professors from their undergraduate years who were near idolised by students. To create this culture in psychiatry would give students considering a career in psychiatry a template of how they can progress. At present, however, psychiatrists are seldom found on the ward, or delivering lectures (a common issue raised by other schools). There is great difficulty even finding psychiatrists to facilitate the psychiatry problem-based learning. The blame for these problems is not confined to one organisation and progress is being made.

Nevertheless, I have enjoyed my psychiatry rotation and have been steeled towards the specialty as a career. It is encouraging to see a more evangelical approach to recruitment being propagated by the Royal College of Psychiatrists, and I look forward to the debate continuing.

Edward R. Fearnley, medical student, Lancaster University, email: e.fearnley@lancaster.ac.uk

\section{doi: $10.1192 / p b .36 .7 .272$}

\section{Driving in a crisis}

We wholeheartedly commend Dr Sheridan on his recent article on fitness to drive ${ }^{1}$ and thank him for highlighting such an important issue.

All drugs acting on the central nervous system can potentially impair alertness, concentration and driving performance. This is particularly so at initiation of treatment, soon after and when dosage is being increased. Driving must cease if adversely affected. Doctors have a duty of care to advise their patients of the potential dangers of adverse effects from medications and interactions with other substances, especially alcohol. The Driver and Vehicle Licensing Agency (DVLA) has published a list of psychiatric conditions and the requirements for notification. Its directives make clear distinction between group 1 drivers (of cars and motorcycles) and group 2 drivers (of lorries and buses). To regain the licence, the DVLA must be satisfied that an improvement in the mental state has been achieved and a period of stability has been fulfilled, which varies for every condition and between groups 1 and $2{ }^{2}$ Crisis resolution teams deal on a daily basis with most of the psychiatric conditions which should be declared to DVLA, such as severe anxiety states or depressive illness, acute psychotic disorders of any type, hypomania/mania, chronic schizophrenia, personality disorders, and substance misuse. In addition, driving can be used as a means of suicide or as a means to harm others, which emphasises the need of a thorough assessment, accurate documentation and regular review. There are a number of incidences such as the tragic event of a mental health service user who lost control behind the wheel killing herself and two members of the public. ${ }^{3}$

I believe the assessment of fitness to drive should be incorporated in day-to-day risk assessment and clearly documented at each contact with crisis team service users. This is core business of every professional who comes in touch with patients. Patients deserve to be advised with regard to DVLA regulations, and indeed should stop driving if deemed unsafe and advised to contact the DVLA accordingly. The General Medical Council advises clinicians to tell patients with conditions which are likely to impair their ability to drive to inform the DVLA. If, however, the clinician does not assess and monitor the particular risk, they would be failing in their statutory duty, irrespective of their need to break confidentiality or not. ${ }^{4}$ 